\title{
Mathematical Modeling of Cervical Cancer with HPV Transmission and Vaccination
}

\author{
Abdulsamad Engida Sado \\ Mathematics Department's, College of Natural and Computational Sciences, Bule Hora University, Bule Hora, Ethiopia \\ Email address: \\ samutiya@gmail.com

\section{To cite this article:} \\ Abdulsamad Engida Sado. Mathematical Modeling of Cervical Cancer with HPV Transmission and Vaccination. Science Journal of Applied \\ Mathematics and Statistics. Vol. 7, No. 2, 2019, pp. 21-25. doi: 10.11648/j.sjams.20190702.13
}

Received: March 1, 2019; Accepted: March 25, 2019; Published: June 26, 2019

\begin{abstract}
Cervical cancer is the most common cancer which affects women next to breast cancer in the worldwide. Most cervical cancers are related to Human Papillomavirus (HPV) infection. We can prevent about 40\% of cancer through Primary prevention and considered as the most cost-effective way of battling cancer. In this paper I have constructed a mathematical model of cervical cancer with Human papilloma virus transmission with and without vaccination. Infection with human papillomavirus (HPV) is the main cause of cervical cancer. To construct the mathematical model I consider the population in four compartment (the number of susceptible women, the number of infected women with HPV. Since HPV infected human may or may not infected by cervical cancer, we have two additional compartments, the number of Infectious HPV women population who are uninfected with cervical cancer and number of Infectious HPV women population who are infected with cervical cancer. Ordinary differential equation is used to construct the model and dimension less model is obtained, then the model validity lucidly verified using simulation study and mathematical analysis tools. These models have generally shown that vaccinating females can be technique to reduce and control cervical cancer. Essential observations are made from the simulation study and physical interpretations are drawn and presented strongly in the paper.
\end{abstract}

Keywords: Cervical Cancer, Mathematical Modeling, Numerical Solution, Human Papillomavirus

\section{Introduction}

The body of all person is a collection of several living cells. Normal body cells growth separate into new cells. At early years of all person's life, normal cells divide faster to allow the person to grow up. After each person becomes an adult, most cells are divided only to replace dying cells, or to repair damage cells. Naturally cells in the body born and die. In mandate to balance natural death of the cell and to maintain cell population equilibrium state, the normal cell in human body divide themselves and grow in number. Uncontrolled growth of abnormal cells is a result of a set of mutations in the healthy cells. Cancer rises from unbounded and fast growth of cell takes place in human body. As a whole, cancer is a collection of more than one hundred diseases in human body [1-4]. A range of cancer disease can occur in human body. In general, name to the cancer disease is given from the name where affected by cancer disease: for example breast cancer, cervical cancer, Brain cancer, Lung cancer, Blood cancer, Bone cancer are few in the list. Slightly any party of the human body can be affected by cancer [2-3].

Cervical cancer is the most common cancer which affects women next to breast cancer in the worldwide. Most cervical cancers are related to Human Papillomavirus (HPV) infection. There are more than 100 different kinds of HPV, most of them are safe. Nevertheless, some types of HPV can disturb the normal functioning of the cervix cells. There are many types of HPV: for example HPV-16, HPV-18, HPV-31, HPV-35, HPV-39, HPV-45, HPV-51, HPV-52, and HPV-58 are some of them. About $70 \%$ of all cervical cancers are caused by HPV-16 and HPV-18 [2-3].

The symptoms of cervical cancer are pain during sexual intercourse, unusual vaginal bleeding, increased amount of discharge and foul-smelling discharge from vagina. Cervical cancer is an abnormal kind of cancer that develops in woman's cervix; it is the entrance to the womb from vagina $[1-2,4]$. The cervix links the uterus to the vagina. Blood flows from the uterus through the cervix into the vagina during a menstrual period. Cervical cancer may sometimes be a danger to life. It can attack nearby tissues and organs. It 
can spread to other parts of the body. Cervical cancer cells can spread by breaking away from the cervical tumor. They can transmit through lymph vessels to nearby lymph nodes. The spread of cancer cells can also occurred through the blood vessels to the bones, liver or lungs [4].

In Ethiopia, cancer records for about $5.8 \%$ of total national mortality (Globocan 2012). Although population-based data do not exist in the country except for Addis Ababa, it is estimated that the annual incidence of cancer is around 60,960 cases and the annual mortality is over 44,000. In Ethiopia, patients often present with advanced stages of cancer. Over $80 \%$ of deaths from non-communicable diseases are caused by four main diseases such as cardiovascular disorders, cancer, diabetes mellitus and chronic obstructive pulmonary disease. These four major non-communicable diseases share similar risk factors.

The first Ethiopian National Cancer Control is bring into line with the priorities of the National Health Sector Transformation Plan (HSTP) 2015/16 - 2019/20 of the Federal Democratic Republic of Ethiopia, and recommends a comprehensive cancer control strategy and interventions with estimates of the cost required to deliver the plan over five years. The plan outlines interventions to reduce the burden of cancer through changes in lifestyle, primary prevention, screening and early diagnosis, appropriate follow-up, treatment and provision of palliative care. The most prevalent cancers in Ethiopia among the entire adult population are, breast cancer $(30.2 \%)$, cancer of the cervix $(13.4 \%)$ and colorectal cancer (5.7\%). About two-thirds of annual cancer deaths occur among women (AACR 2014).

Factors and Prevention of cancer: According to Globocan 2012 estimates, about $40 \%$ of cancers are preventable. The risk factors for cancer are profoundly associated with socioeconomic status; they are higher for populations with lowsocio-economic-status populations, where cancer survival is lower than in wealthier social settings. The risk factors for cancer can be broadly categorized into four types, namely behavioral risk factors, biological risk factors, environmental risk factors and genetic risk factors. Behavioral risk factors include tobacco use, harmful use of alcohol, unhealthy diet and physical inactivity.

Biological factors include overweight, obesity, age, sex of the individual and Genetic/hereditary make up. Environmental risks include exposure to environmental carcinogens such as chemicals, radiation and infectious agents (including certain viruses) [2, 10-14].

Cancers are preventable through interventions such as tobacco control, promotion of healthy diets and physical activity, protection against exposure to environmental carcinogens and vaccination against specific infections. We can prevent about $40 \%$ of cancer through Primary prevention and considered as the most cost-effective way of battling cancer. $[4,14]$

\section{Mathematical Model of Cervical Cancer}

Mathematical modeling is the computational language change the real world problem to mathematical equation. Particularly communicable disease and non-communicable disease modeling now a days popular to predict, control transmission and disease management.

Most cervical cancers are related to Human Papillomavirus (HPV) infection. There are more than 100 different kinds of HPV, most of them are harmless. Nevertheless, some types of $\mathrm{HPV}$ can annoy the normal functioning of the cells of the cervix. About $70 \%$ of all cervical cancers are caused by HPV-16 and HPV-18. We construct the model for cervical cancer by dividing the model into four compartments. These are the number of susceptible women (X1), the number of infected women with HPV (X2). Since HPV infected human may or may not infected by cervical cancer, we have two additional compartments, the number of Infectious HPV women population who are uninfected with cervical cancer (X3) and number of Infectious HPV women population who are infected with cervical cancer (X4). The flow diagram of the model is given as the following

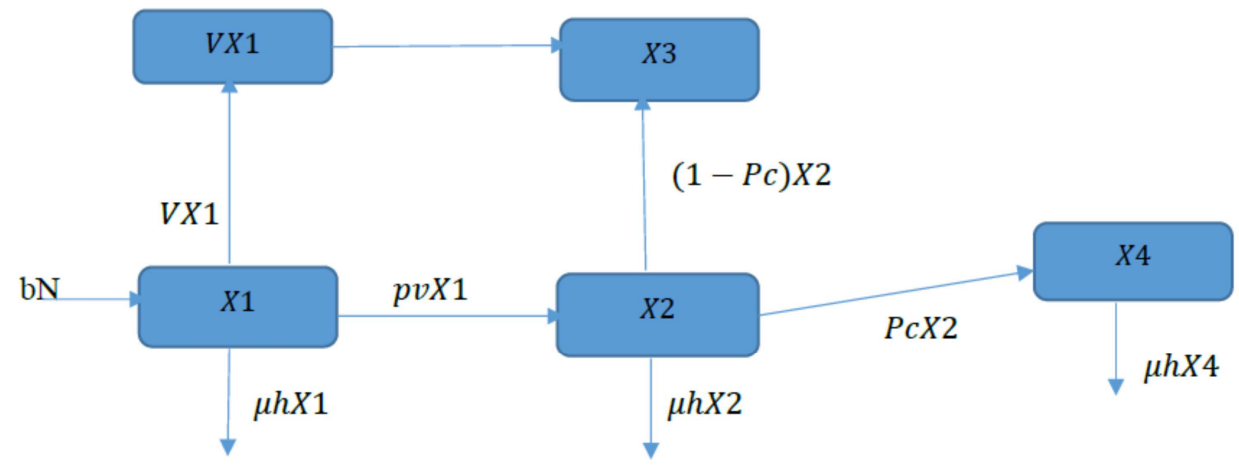

Figure 1. Flow diagram of simple compartment Model.

$$
\begin{gathered}
\frac{d X_{1}}{d t}=b N-P_{v} X_{1}-\mu_{h} X_{1}-v X_{1} \\
\frac{d X_{2}}{d t}=P_{v} X_{1}-\left(1-P_{c}\right) X_{2}-\left(P_{c}+\mu_{h}\right) X_{2}
\end{gathered}
$$

$$
\begin{gathered}
\frac{d X_{3}}{d t}=\left(1-P_{c}\right) X_{2}-\mu_{h} X_{3}+v X_{1} \\
\frac{d X_{4}}{d t}=P_{c} X_{2}-\mu_{h} X_{4}
\end{gathered}
$$


Model assumption

1. Susceptible population all girls and women after 15 years

2. There is natural death and cervical cancer death

3. Vaccinated susceptible population are those take full vaccination before they are infected by HPV

4. Population divides in to four compartment Variable and parameter in the model

\begin{tabular}{|c|c|}
\hline ymbol & Definition \\
\hline$X_{1}$ & number of susceptible women \\
\hline$X_{2}$ & $\begin{array}{l}\text { number of infected women with human } \\
\text { papilloma virus (HPV) }\end{array}$ \\
\hline$X_{3}$ & $\begin{array}{l}\text { number of Infectious HPV women population } \\
\text { who are uninfected with cervical cancer }\end{array}$ \\
\hline$X_{4}$ & $\begin{array}{l}\text { number of Infectious HPV women population } \\
\text { who are infected with cervical cancer }\end{array}$ \\
\hline$b h$ & birth rate of women population, \\
\hline$\mu h$ & death rate of women population \\
\hline$N p$ & total number of women, \\
\hline$v X_{1}$ & $\begin{array}{l}\text { number of women population who obtain } \\
\text { vaccination and develop insusceptibility }\end{array}$ \\
\hline$P v$ & $\begin{array}{l}\text { probability of women who be infected with } \\
\text { human papilloma virus (HPV) }\end{array}$ \\
\hline$p c$ & $\begin{array}{l}\text { robability of women with HPV can be infe } \\
\text { ith cervical cancer }\end{array}$ \\
\hline
\end{tabular}

\subsection{Analysis of the Cervical Cancer Model}

In this section I accomplish analysis of the cervical cancer model designed using dynamics discrete model. The analysis includes (i) dimensionless form of the model, (ii) identifying equilibrium points, (iii) stability analysis of the equilibrium points, and (iv) positivity of equilibrium points. This analysis is mandatory and helpful to handle the parametric values in simulation study so as to obtain biologically meaning full outcomes or conclusions.

\subsubsection{Dimension Less Form}

Dimensionless form of a mathematical model helps (i) to decrease the number of parameters involved in the model and (ii) to make the variables dimensionless, that is, the units of the variables are removed. Note that the minimization of the number of parameters and eliminating the units of variables will make the mathematical analysis simple. Hence, I construct the dimensionless form, the model of equation (1a), (1b), (1c), (1d) in this section as follows:

Now I introduce four dimensionless variables, $x_{1}, x_{2}, x_{3}$, and $x_{4}$, defined by $x_{1}=\frac{X_{1}}{N}, x_{2}=\frac{X_{2}}{N}, x_{3}=\frac{X_{3}}{N}$ and $x_{4}=\frac{x_{4}}{N}$ then by substituting these new dimension less variable to the model in equation (1), (2), (3), (4) and simplify, I obtain the following dimension less model.

$$
\begin{gathered}
\frac{d x_{1}}{d t}=\mu_{h}\left(1-x_{1}\right)-\left(P_{v}+v\right) x_{1} \\
\frac{d x_{2}}{d t}=P_{v} x_{1}-\left(1+\mu_{h}\right) x_{2} \\
\frac{d x_{3}}{d t}=\left(1-P_{c}\right) x_{2}-\mu_{h} x_{3}+v x_{1}
\end{gathered}
$$

$$
\frac{d x_{4}}{d t}=P_{c} x_{2}-\mu_{h} x_{4}
$$

From the dimension less form of constructed cervical cancer model given in equation (5), (6), (7), (8), now identify the equilibrium points and undertake the stability analysis of these equilibrium points as follows:

\subsubsection{Equilibrium Points}

A point $x^{*}$ in real line $R$ is said to be an equilibrium point of one dimensional ordinary differential equation $x^{\prime}=f(x)$ if the point satisfies the condition $f\left(x^{*}\right)=0$. Equilibrium point can also be termed as stationary point. Now in order to obtain the stationary point of the model equation (5), (6), (7), (8) let us set the right hand sides of each of the four equations in (5), (6), (7), (8) simultaneously equal to 0 and solve, so as to get the following:

$$
\begin{gathered}
x_{1}{ }^{*}=\frac{\mu h}{\mu h+p_{v}+v} \\
x_{2}{ }^{*}=\frac{p_{v} \mu h}{(\mu h+p v+v)(1+\mu h)} \\
x_{3}{ }^{*}=\frac{(1-p c) p_{v}+v(1+\mu h)}{(\mu h+p v+v)(1+\mu h)} \\
x_{4}{ }^{*}=\frac{p_{c} p_{v}}{(\mu h+p v+v)(1+\mu h)}
\end{gathered}
$$

The dimensionless model (2) has four equilibrium point $P=\left(x_{1}{ }^{*}, x_{2}{ }^{*}, x_{3}{ }^{*}, x_{4}{ }^{*}\right)$ where the expression for $x_{1}{ }^{*}, x_{2}{ }^{*}, x_{3}{ }^{*}, x_{4}{ }^{*}$ are described in (9), (10), (11), (12). Now, take up the analysis of the stability of this equilibrium point $P=\left(x_{1}{ }^{*}, x_{2}{ }^{*}, x_{3}{ }^{*}, x_{4}{ }^{*}\right)$ by (i) constructing the Jacobian matrix and (ii) finding the eigenvalues of the Jacobian matrix and (iii) finding the signs of these Eigen values at the equilibrium point.

\subsubsection{Positivity of Equilibrium Point}

In order to show that the model (5), (6), (7), (8) is well defined and is biologically meaning full, we restrict that all of the four compartment population sizes can approach a non-negative steady state. Hence, we establish the condition on the parameters as $\mathbb{R}_{+}^{4}=\left\{\left(x_{1}, x_{2}, x_{3}, x_{4}\right): x_{1}, x_{2}, x_{3}, x_{4} \geq\right.$ $0\}$. These non-negativity conditions are required to be satisfied for the equilibrium points. To guarantee the $x_{1}, x_{2}, x_{3}, x_{4} \geq 0$, for all value of the parameters $\mu h, p_{v}, p_{c}, v \geq 0$ found in the dimension less form of model is positive, hence, the mathematical model of cervical cancer model is well defined both mathematically and biologically.

\subsection{Stability Analysis of the Equilibrium Points}

The Jacobian matrix is used to analyze the local stability of equilibrium points where the Jacobian matrix for the model equation (5), (6), (7), (8) is given by;

$$
J=\left[\begin{array}{cccc}
-\mu h-p_{v}-v & 0 & 0 & 0 \\
p_{v} & -(1+\mu h) & 0 & 0 \\
v & 1-p_{c} & -\mu h & 0 \\
0 & p_{c} & 0 & -\mu h
\end{array}\right]
$$

and the Jacobian matrix at the equilibrium point $P=$ 
$\left(x_{1}{ }^{*}, x_{2}{ }^{*}, x_{3}{ }^{*}, x_{4}{ }^{*}\right)$ takes the form

$$
\left[\begin{array}{cccc}
-\mu h-p_{v}-v & 0 & 0 & 0 \\
p_{v} & -(1+\mu h) & 0 & 0 \\
v & \left.1-x_{c}{ }^{*}, x_{2}{ }^{*}, x_{3}{ }^{*}, x_{4}{ }^{*}\right)= & -\mu h & 0 \\
0 & p_{c} & 0 & -\mu h
\end{array}\right] \text { (14) }
$$

The characteristic equations of matrices

$$
\left(\lambda+\mu h+p_{v}+v\right)(\lambda+(1+\mu h))(\lambda+\mu h)(\lambda+\mu h)=0
$$

Hence, the four Eigen values of the Jacobian matrices evaluated at the equilibrium point are given by

$$
\begin{gathered}
\lambda_{1}=-\left(\mu h+p_{v}+v\right) \\
\lambda_{2}=-(\lambda+\mu h) \\
\lambda_{3}=-\mu h \\
\lambda_{4}=-\mu h
\end{gathered}
$$

Based on the algebraic sign of the Eigen values $\lambda_{1}, \lambda_{2}, \lambda_{3}$ and $\lambda_{4}$ of the Jacobian matrix, we can determine the nature of the equilibrium points. Since all the Eigen values are less than zero (negative) the dimensionless system (5), (6), (7), (8), describe cervical cancer is stable at the equilibrium point

$$
P=\left(x_{1}{ }^{*}, x_{2}{ }^{*}, x_{3}{ }^{*}, x_{4}{ }^{*}\right)
$$

\section{Numerical Analysis for the Constructed Cervical Cancer with HPV}

This numerical solution is the solution solved by using ode45 solver (embedded Runge -Kuta 45) of Matlab R2013a to obtain high quality results and relatively fast. The following figure 2, is plot of cervical cancer and HPV transmission from one compartment to another compartment. The vaccine is not applied to the model or $v=0$.

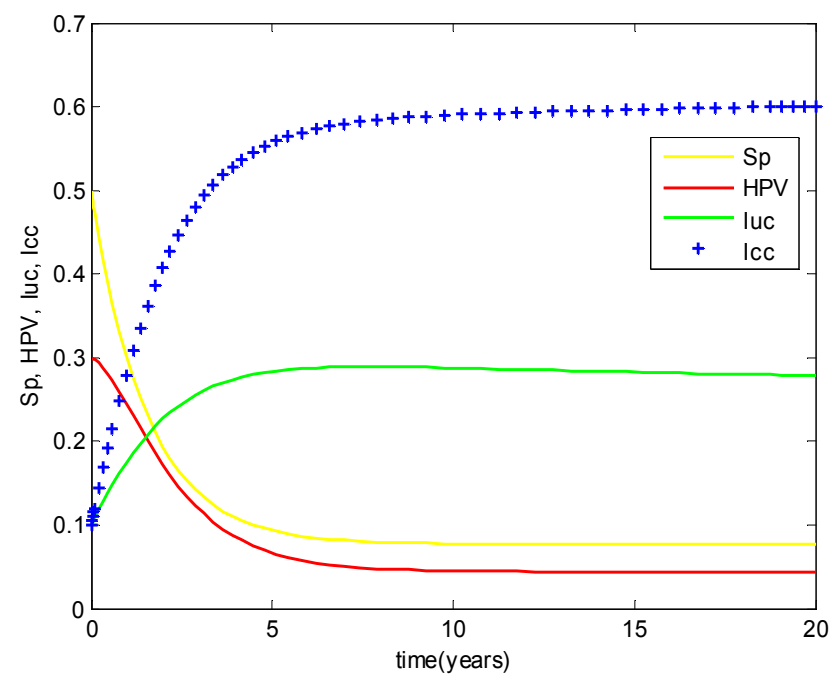

Figure 2. Transmission of HPV and Cervical cancer without Vaccination $(v=0)$.
In this graph, the number of susceptible population is decrease quickly below 0.1 , since the chance of all susceptible population infected by Human papillomavirus is wide. The number of cervical cancer infected population (Icc) are increased from 0.1 to above 0.6 in five years. Also there is a group of population have natural immunity who are infected by HPV but uninfected by cervical cancer (Iuc).

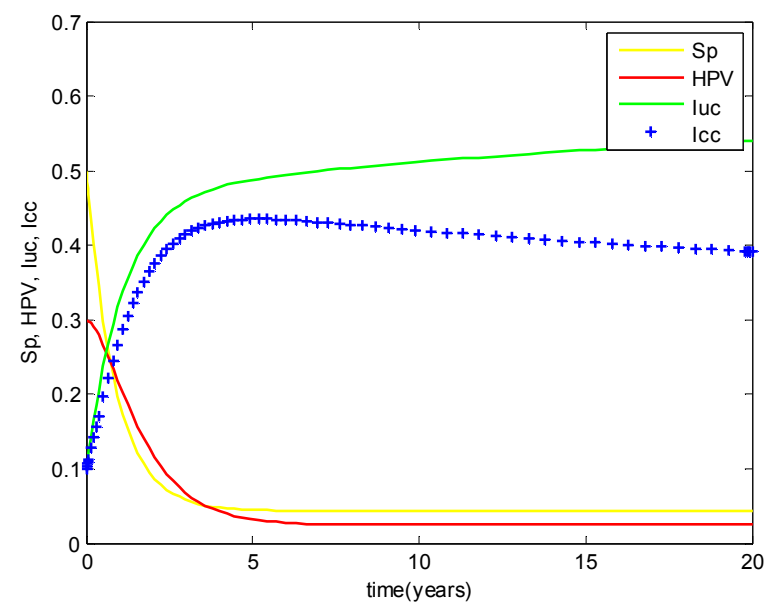

Figure 3. Transmission of HPV and Cervical cancer with Vaccination $(v=0.5)$.

In the figure 3, vaccination is given for the half of susceptible population, hence the number of population infected with HPV are decreased, due to this, the number of uninfected cervical cancer are increase and the number of infected cervical cancer decrease.

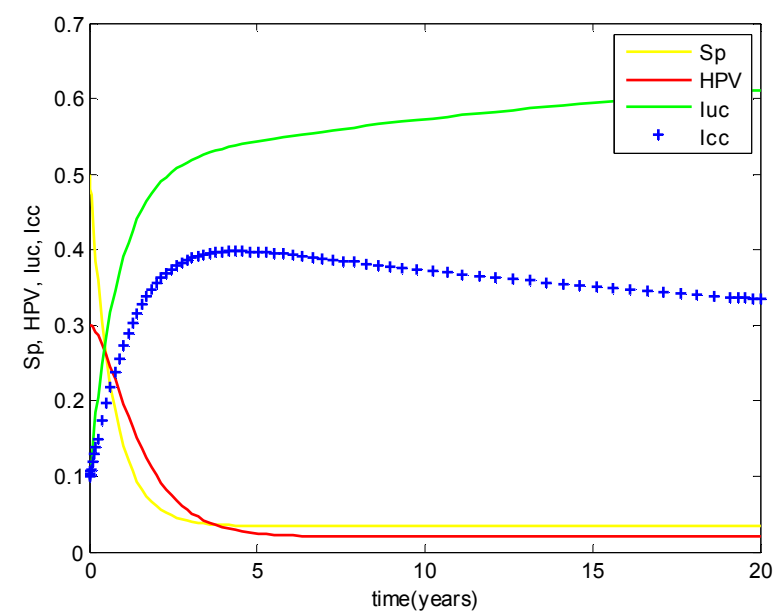

Figure 4. Transmission of HPV and Cervical cancer with Vaccination $(v=0.8)$.

In the figure 4, vaccination is given for the $v=0.8$ of susceptible population, hence, the number of population infected with HPV are decreased very quickly, due to this, the number of uninfected cervical cancer increases rapidly and the number of infected cervical cancer decreases.

In the figure 5 , the susceptible population quickly decrease as the number of vaccination given for the population increases. Population those take the vaccination develop the 
immunity before they are infected by HPV, and the number of cervical cancer infected population decrease as the number of population get vaccination increases. Therefore, vaccination take a great role to control the types of cancer transmitted by HPV.

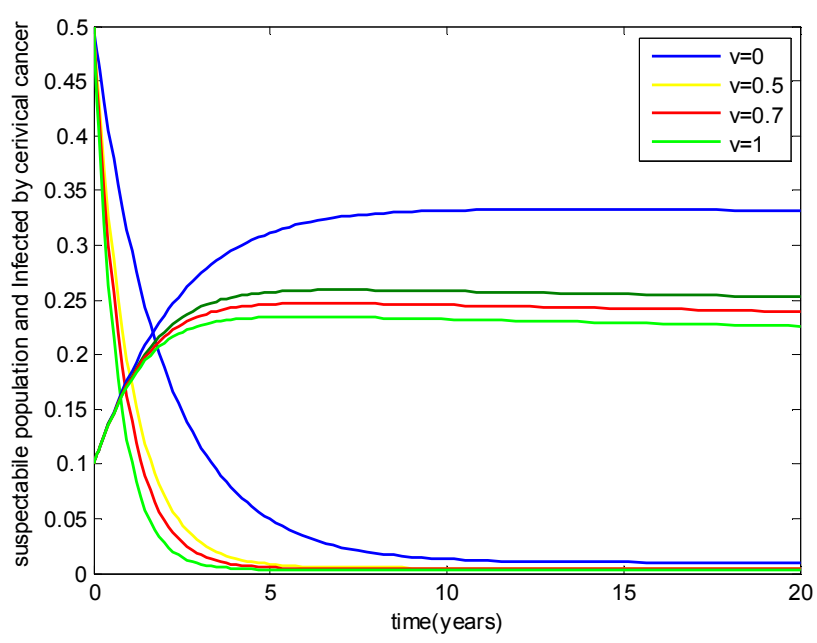

Figure 5. Susceptible population and cervical cancer infected with different value of vaccination.

\section{Conclusion}

Mathematical modeling has been an ever collective tool to sightsee complex systems and unsolved problems in different field of study especially in health science, so as to make the study of their behavior simple. In this paper, I have applied mathematical modeling of cervical cancer transmission by Human papilloma virus with vaccination. In this study I considered population by dividing in to four compartments. Susceptible population, population infected by Human papilloma virus, population those infected by HPV and not infected by cervical cancer due to natural immunity and those infected by virus and infected by cervical cancer. When vaccine is given to a susceptible population, those population develop the immunity from attacking by human papilloma virus which is case $70 \%$ of cervical cancer. As it is observed in the figure 4, the vaccine play great roll to reduce and control the transmission of the human papilloma virus, and cervical cancer. Furthermore, the simulation study supports that the application of mathematical modeling to cervical cancer model is biologically meaning full and mathematically well defined. The model constructed and studied in this paper is local stable at the stationary point.

\section{References}

[1] Aweke YH, Ayanto SY, Ersado TL (2017) Knowledge, attitude and practice for cervical cancer prevention and control among women of childbearing age in Hossana Town, Hadiya, zone, Southern Ethiopia: Community-based cross-sectional study. PLoS ONE 12 (7): e0181415.
[2] Netsanet AA. Cervical Cancer: Ethiopia's Outlook. J Gynecol women's Health. 2017; 5 (2): 555660. DOI: 10.19080/JGWH.2017.05.555660.

[3] Abdulsamad Engida Sado, Purnachandra Rao Koya. Application of Brody Growth Function to Describe Dynamics of Breast Cancer Cells. American Journal of Applied Mathematics. Vol. 3, No. 3, 2015, pp. 138-145. doi: 10.11648/j. ajam.20150303.20.

[4] Federal ministry of health, Ethiopia, Disease prevention and control directorate, national cancer control plan 2016-2020, October 2015.

[5] Lucy W Kivuti-Bitok, Ganesh P Pokhariyal, Geoff McDonnell, Roudsari Abdul. A Mathematical Model of Cervical Cancer in Kenya. International Journal of Science and Research (IJSR). Volume 4 Issue 2, February 2015, ISSN (Online): 2319-7064.

[6] Erik J. Dasbach, Elamin H. Elbasha, and Ralph P. Insinga. Mathematical Models for Predicting the Epidemiologic and Economic Impact of Vaccination against Human Papillomavirus Infection and Disease, Vol. 28, 2006, DOI: 10.1093/epirev/mxj006, Advance Access publication June 1, 2006.

[7] Mesfin Tafa Segni, Hailu Fekadu Demissie, Dagne Mulu Tadesse, Roza Amdemichael, Yonas Teshome Mamo, Amare Worku. Level of Knowledge and Associated Factor toward Cervical Cancer among Women Age (21-64) Years Visiting Health Facilities in Gulele, Sub-city Addis Ababa Ethiopia, JOP. J Pancreas (Online) 2017 Jan 25; 18 (1): 44-48.

[8] Mathematical analysis for modeling, Joudah Rosenbalt; with collaboration of Stoughton Bell.

[9] D M Parkin, S M Moss. An evaluation of screening policies for cervical cancer in England and Wales using a computer simulation model, Journal of Epidemiology and Community Health, 1986, 40, 143-153.

[10] Sue J. GOLDIE, Daniel GRIMA, Michele Kohthomas c. wright, Milton Weinsteinand Eduardo Franco, A Comprehensive Natural History Model Of HPV Infection And Cervical Cancer to Estimate The Clinical Impact Of A Prophylactic Hpv-16/18 Vaccine.

[11] Barnabas RV, Laukkanen P, Koskela P, Kontula O, Lehtinen M, et al. (2006) Epidemiology of HPV 16 and cervical cancer in Finland and the potential impact of vaccination: Mathematical modelling analyses.

[12] Moscicki A, Hills N, Shiboski S, et al. Risks for incidence human papillomavirus infection and low-grade squamous intraepithelial lesion development in young females. JAMA 2001; 285: 2995-3002.

[13] Manhart LE, Koutsky LA (2002) Do condoms prevent genital HPV infection, external genital warts, or cervical neoplasia? A meta-analysis. Sex Transm Dis 29: 725-735.

[14] What you need to know about Cervical cancer, National Cancer Institute, US. Department of health and human services, National Institute of Health.

[15] Lowy DR, Schiller JT. Papillomaviruses: prophylactic vaccine prospects. Biochimica et Biophysica Acta 1998; 1423: M1M8. 1998. 